\title{
Earth's seven sisters
}

Snellen I.A.G. (2017), Astronomy: Earth's seven sisters, Nature (1476-4687) 542: 421-423. https://doi.org/10.1038/542421a 


\section{Earth's seven sisters}

Seven small planets whose surfaces could harbour liquid water have been discovered around a nearby dwarf star. If such a configuration is common for planetary systems, our Galaxy could be teeming with Earth-like planets. See Letter p.xxx

\section{Ignas A. G. Snellen}

Most stars in the Milky Way are much smaller and dimmer than our Sun. Low-mass stars that are about 80 times $^{1}$ heavier than Jupiter have core temperatures that are just high enough to convert hydrogen into helium. Consequently, these stars are less than 1,000 times as bright as the Sun. An example of such a star is TRAPPIST-1, which is located only 12 parsecs (39 light years) away ${ }^{2}$. Last year, Gillon et $a l^{2}$ announced the detection of three Earth-sized planets around TRAPPIST-1. On page $\mathrm{xxx}$, the authors ${ }^{3}$ report that the star in fact has seven planets, all of which have masses and sizes comparable to those of Earth.

In the past decade, thousands of planets beyond our Solar System have been discovered using a method called transit photometry. When a planet passes in front of (transits) its host star, a tiny amount of stellar light is blocked, exposing the planet and providing information about its size. Low-mass stars are good targets to search for Earth-sized planets because a larger fraction of the stellar surface is blocked during a transit.

Gillon and colleagues therefore began by monitoring the smallest stars in the vicinity of the Sun, using a dedicated 60-centimetre robotic telescope called TRAPPIST (the Transiting Planets and Planetesimals Small Telescope) in Chile. After their initial TRAPPIST-1 discovery ${ }^{2}$, the authors carried out intense ground-based observations of the star, in addition to 20 days of continuous monitoring using the NASA Spitzer Space Telescope. In the current paper, Gillon et al. present evidence for more than 30 transits, which they attribute to a total of seven planets (Fig. 1).

The authors results' show that the TRAPPIST-1 system is extremely compact, flat and orderly. The six inner planets have orbital periods between 1.5 and 13 days that are all 'near-resonant' — in the same time that the innermost planet makes eight orbits, the second, third and fourth planets revolve five, three and two times around the star, respectively. Such an arrangement causes the planets to exert periodic gravitational influences on one other. This effect results in small shifts in the observed transit times, which the authors used to estimate the planets' masses.

The planetary system is strikingly reminiscent of Jupiter and its Galilean moons, albeit scaled up in mass by a factor of about 80. Io, Europa, Ganymede and Callisto orbit Jupiter with periods between 1.6 and 17 days, also in near-resonance. This resemblance suggests that the TRAPPIST-1 planets and the Galilean moons formed and evolved in a similar way ${ }^{4}$.

In the past few years, evidence has been mounting ${ }^{5,6}$ that Earth-sized planets are abundant throughout the Galaxy, but Gillon and collaborators' findings indicate that these planets are even more common than previously thought. Geometric arguments tell us that for every transiting planet found, there should exist a multitude of similar planets (20 to 100 times more) that, seen from Earth, never pass in front of their host star. Of course, the authors could have been lucky, but finding seven transiting Earth-sized planets in such a small sample suggests that the Solar System with its four (sub-)Earth-sized planets might be nothing out of the ordinary.

Gillon et al. will soon step up their search for planets around the smallest stars with the project SPECULOOS $^{7}$ (Search for Habitable Planets Eclipsing Ultra-cool Stars), which will use four 1metre telescopes and increase the authors' sample of stars by a factor of 10. In addition, NASA plans to launch TESS (the Transiting Exoplanet Survey Satellite), a space telescope that will spend two years identifying planets around more than 200,000 of the brightest stars, including about 10,000 small dwarf stars ${ }^{8}$. Although none of the stars monitored by TESS will be as small as TRAPPIST-1, the higher precision obtained in space will compensate for the weaker photometry signals and allow populations of Earth-sized planets around such stars to be accurately mapped. 
Excitingly, we might soon find out what it is like on these seven sisters of planet Earth. The James Webb Space Telescope, scheduled for launch next year, will be able to detect atmospheric species and thermal emission from the planets ${ }^{9}$, constraining their composition and climate, respectively. Liquid water could possibly exist or have existed on any of the TRAPPIST-1 planets ${ }^{10}$, but predictions are difficult to make. For instance, although the estimated densities of the planets are consistent with them being similar in composition to Earth, they could instead be volatile-rich containing a large fraction of water and ices - like the Galilean moons.

Jupiter's moons also teach us that a crucial factor for predicting the climate of a planetary body is tidal heating - the heating of the interior of a planet due to friction caused by tides. Such an effect is responsible for widespread volcanism on Io and the presence of a subsurface ocean on Europa. Tidal heating is expected for the TRAPPIST-1 planets because they exist in near-resonant orbits.

Could any of the planets harbour life? We simply do not know. One thing is sure, in a few billion years, when the Sun has run out of fuel and the Solar System has ceased to exist, TRAPPIST-1 will still only be an infant star. It burns hydrogen so slowly that it will live on for another tenthousand billion years ${ }^{11}$ - 1000 times longer than the current age of the Universe. Maybe enough time for life to evolve?

Ignas A. G. Snellen is at the Leiden Observatory, Leiden University, 2300 RA Leiden, Netherlands. e-mail: snellen@strw.leidenuniv.nl

1. $\quad$ Kumar, S.S., Astrophys. J. 137, 1121-1125 (1963)

2. Gillon, M. et al. Nature 533, 221-224 (2016).

3. Gillon, M. et al. Nature $\mathbf{x x x}, \mathrm{xxx}-\mathrm{xxx}(2017)$.

4. $\quad$ Goldreich, P. \& Tremaine, S. Astrophys. J. 241, 425-441 (1980).

5. $\quad$ Berta, Z. K., Irwin, J. \& Charbonneau, D. Astrophys. J. 775, 91-102 (2013).

6. $\quad$ Dressing, C. D. \& Charbonneau, D. Astrophys. J. 807, 45-67 (2015).

7. http://www.orca.ulg.ac.be/SPECULOOS/Speculoos main/Home.html.

8. $\quad$ Sullivan, P., et al., Astrophys. J. 809, 77-105 (2015)

9. de Wit, J. et al., Nature 537, 69-72 (2016).

10. Leconte, J. et al., Astron. \& Astrophys. 554, A69 (2013).

11. Laughlin, G., Bodenheimer, P., Adams, F. C., Astrophys J. 482, 420-432 (1997)

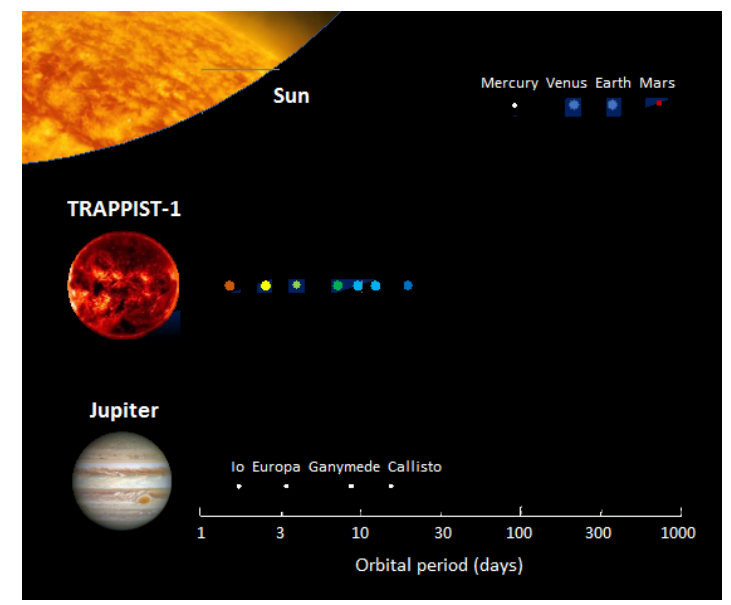

Figure 1 The TRAPPIST-1 planetary system. Gillon et $a .^{2}$ have discovered seven Earth-sized planets in orbit around the nearby dwarf star TRAPPIST-1. Shown here are the measured orbital periods of the planets, compared to those of Jupiter's Galilean moons and the four inner planets of the Solar System. The sizes of all the objects are approximately to scale. 